\title{
Electromagnetics
}

\section{Reducing the Dispersion Errors of the Finite-Difference Time-Domain Method for Multifrequency Plane-Wave Excitations}

\section{UĞUR OĞUZ}

To cite this article: UĞUR OĞUZ (2003) Reducing the Dispersion Errors of the Finite-Difference Time-Domain Method for Multifrequency Plane-Wave Excitations, Electromagnetics, 23:6, 539-552, DOI: $10.1080 / 02726340390222062$

To link to this article: http://dx.doi.org/10.1080/02726340390222062

曲 Published online: 21 Jun 2010.

Submit your article to this journal $\widetilde{ }$

Џ Article views: 15

Q View related articles $\asymp$

4 Citing articles: 2 View citing articles 


\title{
Reducing the Dispersion Errors of the Finite-Difference Time-Domain Method for Multifrequency Plane-Wave Excitations
}

\author{
UĞUR OĞUZ \\ LEVENT GÜREL \\ Department of Electrical and Electronics Engineering \\ Bilkent University \\ Bilkent, Ankara, Turkey
}

\begin{abstract}
We demonstrate the applications of discrete-time signal-processing (SP) techniques for the purpose of generating accurate plane waves in the finite-difference time-domain (FDTD) method. The SP techniques are used either to reduce the high-frequency content of the source excitation or to compute more precise incident-field values in the computational domain. The effects of smoothing windows of various lengths, digital lowpass filters of various bandwidths and characteristics, and polynomial interpolation schemes of various orders are investigated. Arbitrary signals with multifrequency content are considered.
\end{abstract}

Keywords finite-difference time-domain method, plane-wave excitation, numerical dispersion, sampling, windowing, digital filters

\section{Introduction}

Due to its ability to handle multifrequency signals, the finite-difference time-domain (FDTD) method (Yee, 1966) has been widely used to solve scattering problems involving user-defined excitations that introduce high-frequency components to the FDTD grid. However, applying a multifrequency source signal induces a large dispersion error in the FDTD grid. Dispersion-optimization schemes are based on matching the numerical and theoretical phase velocities at a certain (in general, central) frequency component, and the deviations of the numerical phase velocities of all nonzero frequency components increase the error level significantly, when compared to the error level produced by a single-frequency source signal.

In this article, we will demonstrate the application of some signal-processing (SP) techniques, such as filtering, windowing, decimation, and interpolation, to reduce the dispersion errors due to multifrequency plane-wave source functions. These errors can

Received 3 November 2001; accepted 30 June 2002.

This work was supported by the Bilkent University Research Fund EE-01-01 and by the Turkish Academy of Sciences in the framework of the Young Scientist Award Program (LG/TUBAGEBIP/2002-1-12). Ugur Oguz was with Bilkent University at the time this article was written and is now with Aselsan, Inc., Ankara, Turkey.

Address correspondence to Levent Gürel, Dept. of Electrical \& Electronics Engineering, Bilkent University, TR-06800, Bilkent, Ankara, Turkey. E-mail:Igurel@ieee.org 
be observed in the form of nonphysical DC offsets, signal distortions, and added noise (Umashankar \& Taflove, 1982; Taflove \& Umashankar, 1983; van Gennip, 1992; Furse, Mathur, \& Gandhi, 1990). In Gürel and Oğuz (2000), Oğuz, Gürel, and Arıkan (1998), and Oğuz and Gürel (1997), the applications of the aforementioned SP techniques were demonstrated for FDTD computations with sinusoidal excitations. This article shifts the focus to the application of the SP techniques to transient signals with arbitrary spectra.

\section{Excitation of the FDTD Grid}

When the FDTD formulation of an electromagnetic scattering problem requires a source that radiates from a far-zone point, it is possible to simulate the incident waves in the computational domain by defining equivalent sources on a closed surface around the scatterer (Merewether, Fisher, \& Smith, 1980). Such an equivalent-source scheme can be implemented using the total-field formulation in the FDTD method (Taflove, 1995). Totalfield formulation yields the separation of the computational domain into two regions, namely the total-field region and the scattered-field region, as illustrated in Figure 1. In the scattered-field region, the free-space scattered-field equations are implemented, while in the total-field region, the total-field variables are computed. The scattered-field and total-field regions are connected by a mathematical boundary, on which a special set of connecting equations are used to introduce the incident fields. The incident-field values are used in the connecting equations to relate the two different types of variables across the total-field/scattered-field interface.

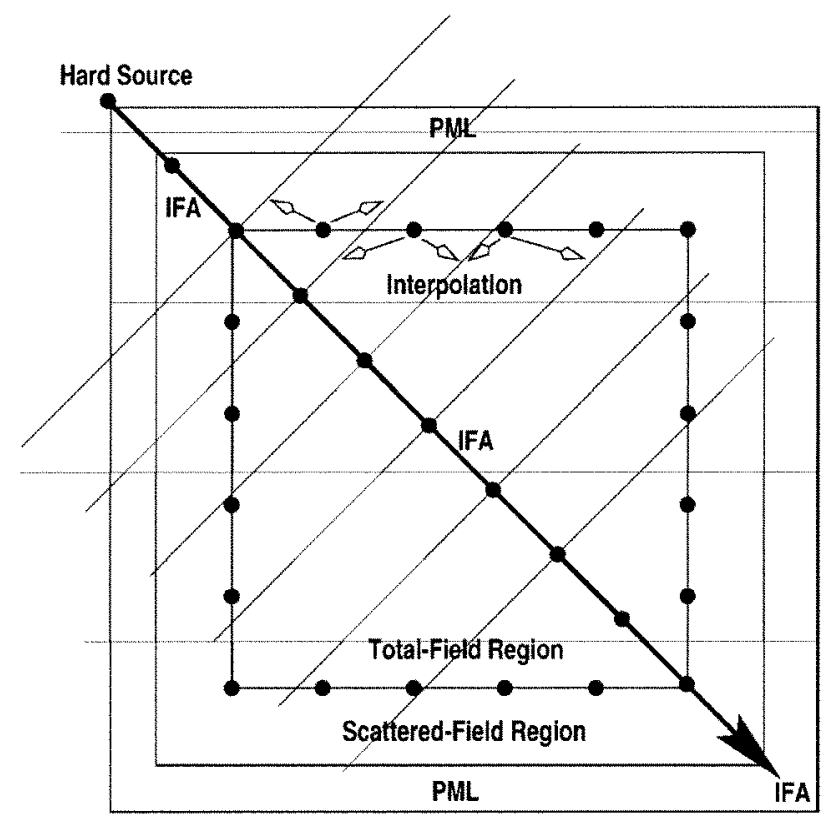

Figure 1. Separated regions in a scattering problem and the plane-wave excitation scheme in the total-field formulation. The scattered-field region is between the total-field region and the ABC. The 1-D source grid points in the direction of propagation, which is arbitrary. The incident-field values in the computational domain are interpolated from the two closest elements of the 1-D source grid (when linear interpolation is used). 
Since the components of the incident field are predefined quantities, it is possible to use closed-form expressions in the connecting equations. The implementation of these equations is simple, but requires the computation of a very large number of complicated terms, such as sinusoids or exponentials. As a more efficient method, a one-dimensional (1-D) look-up table, which is excited by a hard source, is used to compute the incidentfield values (Taflove, 1995). The 1-D array, which is displayed in Figure 1, is assumed to point in the direction of propagation of the incident wave in the computational domain. The incident-field values are generated by the 1-D finite-difference equations in the source grid. The required incident-field values on the total-field/scattered-field interface of the 3-D grid are interpolated from the 1-D data. Throughout this article, a polynomial interpolation scheme with various orders will be used for the incident-field computations. The space-sampling and time-sampling periods in the 1-D array are $\Delta$ and $\Delta t$, the same as in the 3-D FDTD problem.

\section{Numerical Dispersion}

The approximate nature of the FDTD method causes the propagation of electromagnetic waves in the computational domain to deviate from the ideal situation. The waves do not propagate with the theoretical phase velocities in the FDTD grid due to the numerical dispersion errors caused by the finite-differencing scheme. Moreover, each frequency component is subject to a different amount of dispersion error, due to the variation of accuracy as the wavelength changes. The numerical dispersion relation for the discretized medium in a 3-D grid is given by Taflove (1995)

$$
\begin{aligned}
{\left[\frac{1}{c \Delta t} \sin \left(\frac{\omega \Delta t}{2}\right)\right]^{2}=} & {\left[\frac{1}{\Delta x} \sin \left(\frac{k_{x} \Delta x}{2}\right)\right]^{2}+\left[\frac{1}{\Delta y} \sin \left(\frac{k_{y} \Delta y}{2}\right)\right]^{2} } \\
& +\left[\frac{1}{\Delta z} \sin \left(\frac{k_{z} \Delta z}{2}\right)\right]^{2}
\end{aligned}
$$

where $k_{x}, k_{y}$, and $k_{z}$ are the $x, y$, and $z$ components of the numerical wavenumber $k$, respectively.

In the 1-D source grid, two of the three components, $k_{x}, k_{y}$, and $k_{z}$, are identically zero and the above relation simplifies to

$$
\left[\frac{1}{c \Delta t} \sin \left(\frac{\omega \Delta t}{2}\right)\right]^{2}=\left[\frac{1}{\Delta} \sin \left(\frac{k \Delta x}{2}\right)\right]^{2} .
$$

Thus, the incident wave is subject to different wavenumbers and propagates with different phase velocities in the 3-D and 1-D grids.

Both (1) and (2) can be solved in an iterative manner. The ratio of the numerical phase velocities of the incident wave in the 1-D and 3-D grids is used to update the material properties of the source grid by changing the permittivity and the permeability of the 1-D grid. Therefore, the update equations

$$
\begin{aligned}
H_{\mathrm{i} n c}^{i+1 / 2, n+1 / 2} & =H_{\mathrm{i} n c}^{i+1 / 2, n-1 / 2}+\frac{\Delta t}{\left[\frac{v_{p}(\theta=0, \phi=0)}{v_{p}(\theta, \phi)}\right] \mu_{0} \Delta}\left(E_{\mathrm{i} n c}^{i, n}-E_{\mathrm{i} n c}^{i+1, n}\right), \\
E_{\mathrm{i} n c}^{i, n+1} & =E_{\mathrm{i} n c}^{i, n}+\frac{\Delta t}{\left[\frac{v_{p}(\theta=0, \phi=0)}{v_{p}(\theta, \phi)}\right] \epsilon_{0} \Delta}\left(H_{\mathrm{i} n c}^{i-1 / 2, n+1 / 2}-H_{\mathrm{i} n c}^{i+1 / 2, n+1 / 2}\right)
\end{aligned}
$$


are employed in the 1-D source grid. Thus, the velocity in the source grid is assured to match the velocity in the 3-D grid. However, this matching is performed for a single frequency component. Since the input excitation contains more than one frequency component, it is not possible to establish a perfect match between the 1-D and 3-D grids. The optimal frequency component for the matched phase velocities is the dominant frequency of the input excitation.

Another issue concerning the solution of the dispersion relation arises when the wavenumber takes on a complex value for a fixed $(\Delta, \Delta t)$ pair above a certain frequency. For example, for the 1-D source grid, the frequency components above a critical value of (Schneider \& Wagner, 1999)

$$
f_{c}=\frac{1}{\pi \Delta t} \sin ^{-1}\left(\frac{c \Delta t}{\Delta}\right)
$$

yield a complex wavenumber, and thus an exponentially decaying wave as it propagates in the grid. The 3-D grid also has its own critical frequency value, depending on the discretization and the propagation angle, where the numerical wavenumber becomes complex. These two values need not coincide with each other, which means that some frequency components that are located between these two critical values experience exponential decay in one of the grids, and not in the other. These differences of propagation in the two grids produce a leakage from the total-field values to the scattered-field values, i.e., an error, independent of the scatterer in the problem.

\section{Relevant Signal-Processing Tools}

A signal with abrupt changes, e.g., a causal signal, which is zero for $t \leq 0$, contains large high-frequency components, independent of the sampling rate. In Gürel and Oğuz (2000), it was shown that these high-frequency components increased the overall dispersion error in the FDTD grid. Therefore, removing the unwanted components in the source signal reduced this error. In Gürel and Oğuz (2000), it was also demonstrated that smoothing windows could be used to taper the discontinuities of the sinusoidal source signals and to reduce the high-frequency components of the signal spectra. Similarly, in order to reduce the dispersion errors, a smoothing window can be applied to a transient pulse containing such discontinuities and the associated unwanted high-frequency components. In this article, the Hanning window, also known as the raised-cosine window, will be used whenever smoothing is applied to a signal.

In Gürel and Oğuz (2000), it was also shown that the goal of suppressing the highfrequency components of the digital sinusoidal signals could also be achieved by using a digital lowpass filter. Application of a linear-phase finite-impulse-response (FIR) lowpass filter (Parks \& Burrus, 1987; Little \& Shure, 1992) can also be useful to reduce the FDTD errors due to transient pulses. The application of such a filter on the digital signal yields the multiplication of the discrete Fourier transforms of the two signals (Oppenheim \& Schafer, 1989).

The sampling frequency of a discrete signal can be decreased or increased by employing decimation or interpolation schemes, respectively. Assume that the already-sampled signal is downsampled (decimated) by a factor of $M$. The resulting lower sampling rate scales the spectrum of the original signal $M$ times wider and may cause aliasing (Oğuz, Gürel, \& Arıkan, 1998; Oppenheim \& Schafer, 1989). In order to prevent the aliasing, a digital lowpass filter should be employed on the signal before the decimation to reduce the bandwidth below $\frac{\pi}{M}$. 
In contrast to the decimation operation, interpolating new values from the existing samples of a signal does not cause aliasing since the band of resolved frequencies increases with the increased sampling rate. However, the accuracy of the new samples is the important issue in the interpolation scheme. If the original sampling is performed at a sufficiently high frequency, then the induced interpolation error may be neglected. In this case, a simple polynomial (Lagrange) interpolation scheme can be applied to calculate new values of the signal. An $n$-point polynomial interpolation yields the approximation of the value of the signal at any time $t$ from the signal's $n$ original values $x\left(t_{1}\right), x\left(t_{2}\right), \ldots, x\left(t_{n}\right)$ located at time instants $t_{1}, t_{2}, \ldots, t_{n}$ by

$$
\begin{aligned}
x(t)= & \frac{\left(t-t_{2}\right)\left(t-t_{3}\right) \cdots\left(t-t_{n}\right)}{\left(t_{1}-t_{2}\right)\left(t_{1}-t_{3}\right) \cdots\left(t_{1}-t_{n}\right)} x\left(t_{1}\right)+\frac{\left(t-t_{1}\right)\left(t-t_{3}\right) \cdots\left(t-t_{n}\right)}{\left(t_{2}-t_{1}\right)\left(t_{2}-t_{3}\right) \cdots\left(t_{2}-t_{n}\right)} x\left(t_{2}\right) \\
& +\cdots+\frac{\left(t-t_{1}\right)\left(t-t_{2}\right) \cdots\left(t-t_{n-1}\right)}{\left(t_{n}-t_{1}\right)\left(t_{n}-t_{2}\right) \cdots\left(t_{n}-t_{n-1}\right)} x\left(t_{n}\right) .
\end{aligned}
$$

\section{Numerical Results}

In the absence of any scatterer, the fields in the total-field region should be exactly the same as the incident plane wave, and the fields in the scattered-field region should be exactly zero. However, due to the approximate nature of the FDTD method, computationally obtained field variables are expected to deviate from their ideal counterparts. The computational and physical transients of the problem cause slowly decaying or resident errors in the FDTD results. The magnitudes of these errors depend on the parameters of the FDTD computations, including the representation of the excitation. Errors resulting from the plane-wave excitation scheme and their remedies will be presented in the following sections.

In order to demonstrate the error level for a transient-pulsed plane wave, a homogeneous FDTD computational domain of $40 \times 40 \times 40$ cells is set up with a total-field region of $28 \times 28 \times 28$ cells. Perfectly-matched layer (PML) (Bérenger, 1994) absorbing boundary conditions (ABCs) are used to terminate the layered FDTD computational domain. The incident plane wave, whose time variation is shown in Figure 2a, is a $z$-polarized rectangular pulse with zero DC offset and a propagation angle of $\theta=90^{\circ}, \phi=45^{\circ}$. The size of the Yee cells is selected as $6.25 \mathrm{~mm}$ and the time step is set at the Courant stability limit. Fourth-order polynomial interpolation is employed for the computation of the incident-field values of the 3-D grid from the 1-D source grid. Figures $2 \mathrm{~b}-2 \mathrm{~d}$ display the maximum error observed on the $z$ component of the electric field, error on $E_{z}$ at a particular point, and the frequency-domain representation of the error in Figure 2c, respectively.

Figure $2 \mathrm{~b}$ demonstrates that the maximum error level is about $0.5 \mathrm{~V} / \mathrm{m}$, which is about $50 \%$ of the amplitude of the incident pulse, and the residual error level is higher than $0.001 \mathrm{~V} / \mathrm{m}$ after the source is turned off. Figure $2 \mathrm{~d}$ demonstrates that the error signal is dominated by the frequency components between $1 \mathrm{GHz}$ and $16.2 \mathrm{GHz}$, where $1 \mathrm{GHz}$ is the center frequency of the source function, and $16.2 \mathrm{GHz}$ is the frequency that the corresponding wavenumber becomes complex in the 1-D source grid (Schneider \& Wagner, 1999). The $1 \mathrm{GHz}$ term is subject to the phase-velocity-matching algorithm described in the section "Numerical Dispersion," by (3) and (4), but the remaining terms, which have large contributions for the transient pulse, propagate with different phase velocities, which produce a large dispersion error as depicted in Figure 2d. 


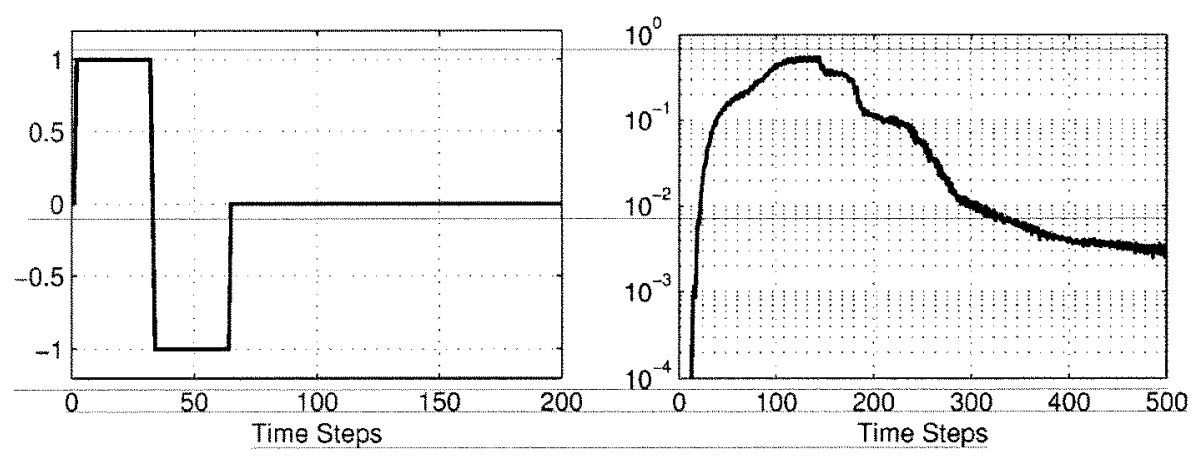

(a)

(b)

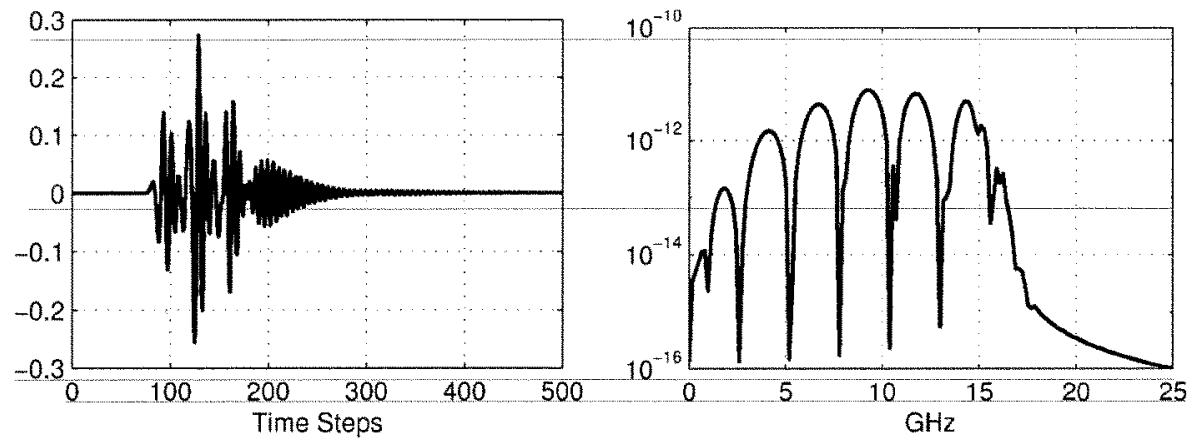

(c)

(d)

Figure 2. Simulation results of a rectangular transient plane wave with a center frequency of $1 \mathrm{GHz}$. (a) Time variation of the source function. (b) Overall maximum error on $E_{z}$. (c) Error signal on $E_{z}$ at a particular point. (d) Frequency-domain representation of the error signal displayed in (c).

\section{Application of Smoothing Windows}

The Hanning window can be applied to the four sharp edges of the source function depicted in Figure 2a. The resultant signal is displayed in Figure 3a. The duration of the pulse and, consequently, the $1 \mathrm{GHz}$ center frequency of the signal are not altered. However, the spectral value of the $1 \mathrm{GHz}$ component of the windowed rectangular pulse is decreased with respect to its rectangular-pulse counterpart. The ratio of the $1 \mathrm{GHz}$ components of the pulses in Figure 2a and 3a is 1.933. However, it is possible to make a quantitative comparison between the error signals obtained with these two source functions, since both signals' time-domain amplitudes are equal to $1 \mathrm{~V} / \mathrm{m}$.

Figures $3 \mathrm{~b}$ and $3 \mathrm{c}$ reveal that the maximum and the typical error levels observed in the FDTD grid are decreased by one order in both the early and late times of the simulation, with respect to the error levels of the rectangular pulse, observed in Figures $2 \mathrm{~b}$ and $2 \mathrm{c}$. The fast Fourier transform (FFT) of the error signal displayed in Figure 3d demonstrates that the smoothing window suppressed the components up to $16 \mathrm{GHz}$.

\section{Application of the Digital Filter}

The digital filter, with frequency response displayed in Figure 4, is applied to the rectangular pulse in Figure 2a, before it is fed into the hard source. The resultant signal is displayed in Figure 5a. 


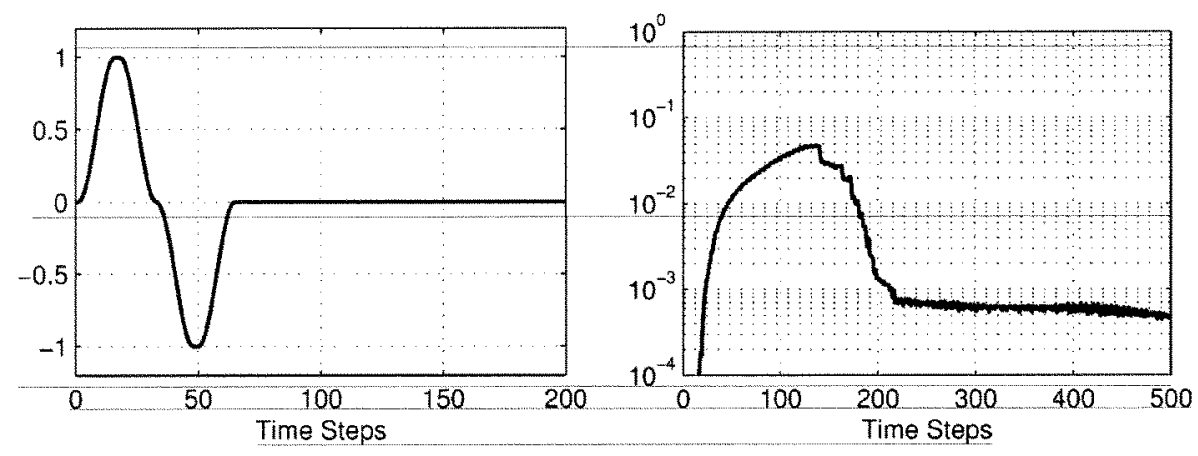

(a)

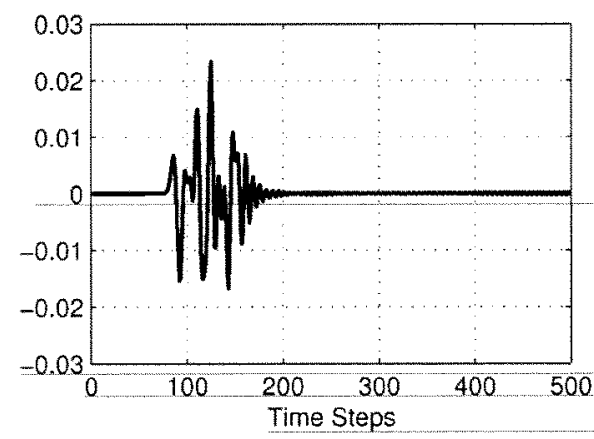

(c) (b)

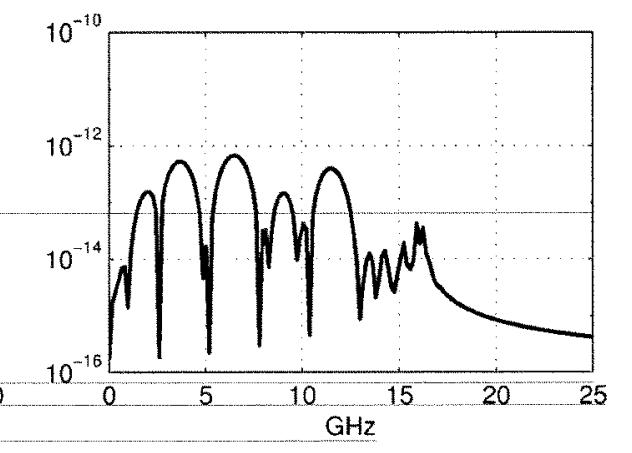

(d)

Figure 3. Simulation results of a windowed transient plane wave with a center frequency of $1 \mathrm{GHz}$. (a) Time variation of the source function. (b) Overall maximum error on $E_{z}$. (c) Error signal on $E_{z}$ at a particular point. (d) Frequency-domain representation of the error signal displayed in (c).

Figure $5 \mathrm{~b}$ reveals that the maximum error level observed in the FDTD grid is decreased by one order in the steady state (where the source is turned off). However, the error level in the transient region is not decreased, since a small portion of the components up to $16.2 \mathrm{GHz}$ are suppressed. The FFT of the error signal shown in Figure 5d also illustrates that the majority of the frequency components of the error signal are unchanged. Since the transition region of the FIR filter is located at the $10-13 \mathrm{GHz}$ band, only the frequencies above $13 \mathrm{GHz}$ are significantly attenuated. Figure 5c suggests that the high-frequency oscillations in the late times of the simulation are diminished, while the early-time error signal is similar in magnitude to its counterpart shown in Fig. 2(c) for the rectangular pulse.

\section{Increasing the Sampling Rate of the 1-D Source Grid}

In Oğuz, Gürel, and Arıkan (1998), it was shown that increasing the resolution of the 1-D source grid decreased the error on the phase velocity of each frequency component in the source function. A transient pulse contains more frequency components compared to a sinusoidal function. Therefore, increasing the sampling rate of the source grid should decrease the overall dispersion error in both 1-D and 3-D grids.

The windowed transient pulse displayed in Figure $3 \mathrm{a}$ is sampled with an 8-timeshigher sampling rate than the ordinary 1-D grid and passed through a 256-point anti- 


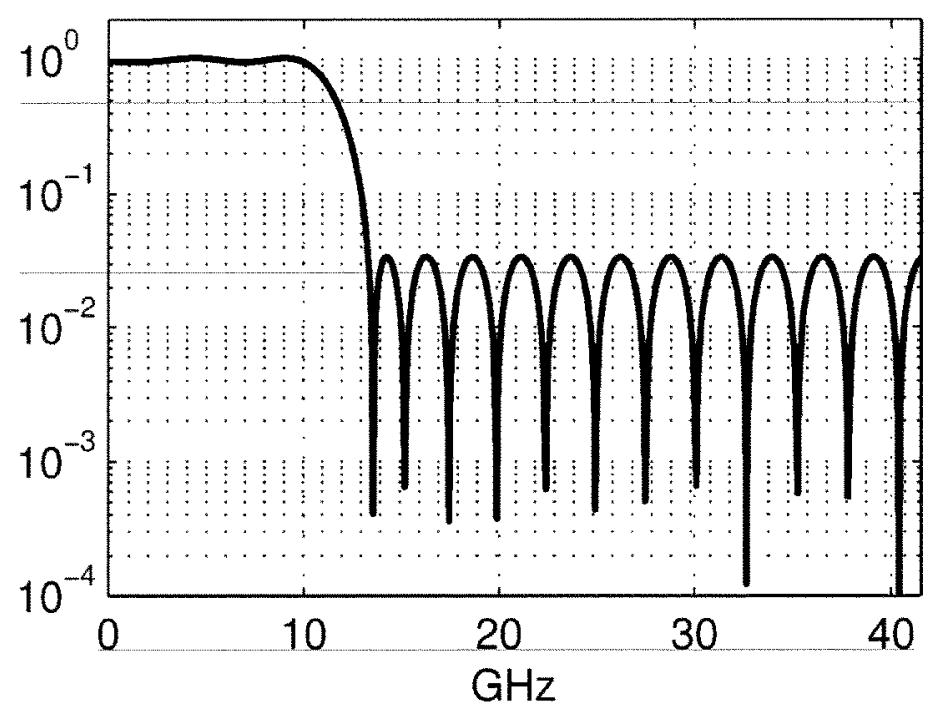

Figure 4. Frequency response of the digital filter used for the suppression of the high-frequency components of the transient plane wave.

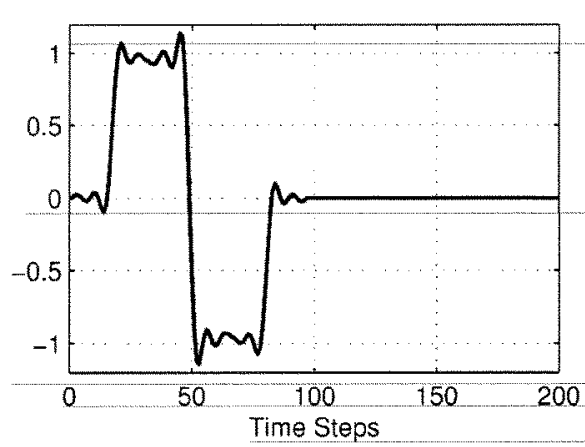

(a)

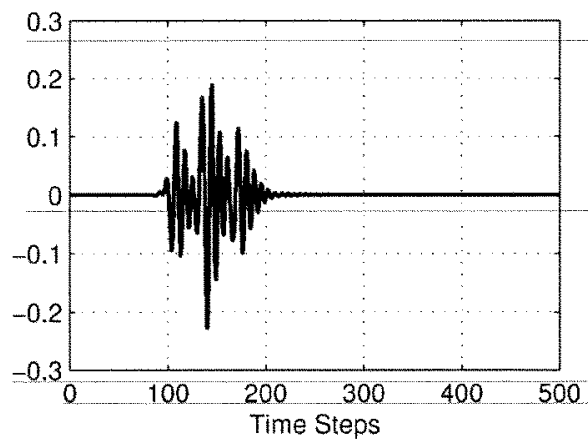

(c)

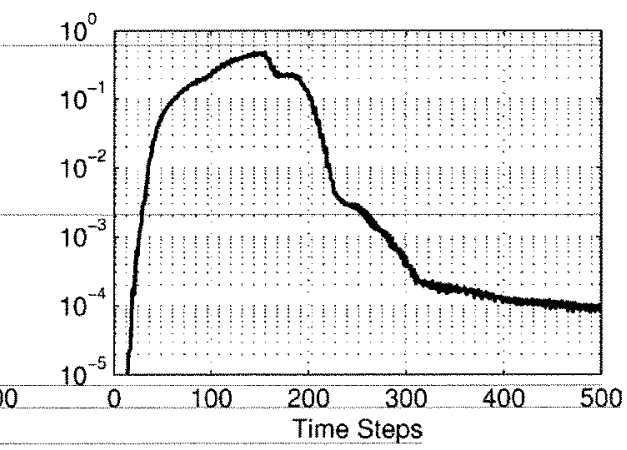

(b)

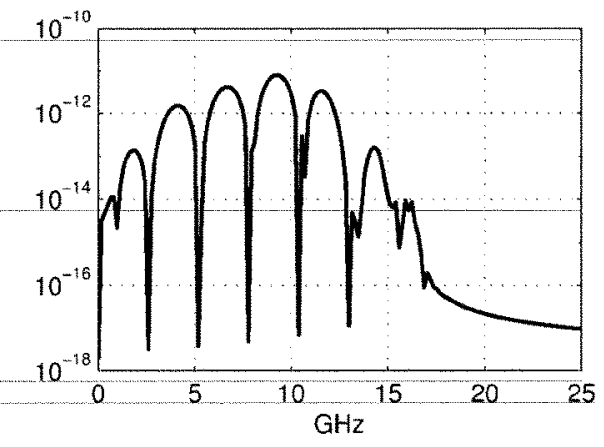

(d)

Figure 5. Simulation results of a filtered transient plane wave with a center frequency of $1 \mathrm{GHz}$. (a) Time variation of the source function. (b) Overall maximum error on $E_{z}$. (c) Error signal on $E_{z}$ at a particular point. (d) Frequency-domain representation of the error signal displayed in (c). 
decimation filter with a cut-off at $\frac{\pi}{8}$ angular frequency to prevent aliasing at the decimation phase. The resultant signal, shown in Figure 6a, is fed into the hard source to propagate in the 1-D source grid. The source grid is operated with a spatial step of $\Delta=0.78125 \mathrm{~mm}$ and a corresponding time step selected at the Courant stability limit. The interpolation scheme for the computation of incident-field values is selected as fourth-order Lagrange. The maximum error on the $z$ component of the electric field is displayed in Figure $6 \mathrm{~b}$. Comparison of Figure $6 \mathrm{~b}$ to $3 \mathrm{~b}$ illustrates an improvement in the error levels in both the transient and the steady-state phases of the simulation. This improvement is better comprehended by observing the decrease in the frequency components of the error signal up to $16.2 \mathrm{GHz}$ for the finer-resolved source-grid result shown in Figure 6d, compared to the error signal of standard source grid displayed in Figure $3 \mathrm{~d}$. The reduction of dispersion error causes an approximately $50 \%$ decrease in the transient error, revealed by the comparison of Figure $6 \mathrm{c}$ to $3 \mathrm{c}$.

The FFT of the error signal displayed in Figure 6d contains frequency components above the critical frequency of the standard source grid, which is explained in the section "Numerical Dispersion." Since the time step of the 1-D source grid is divided by a factor of 8 , the value of the new critical frequency is approximately 8 times larger than its prior value. Therefore, for the frequency values between 0 and $41.5 \mathrm{GHz}$, the wavenumber

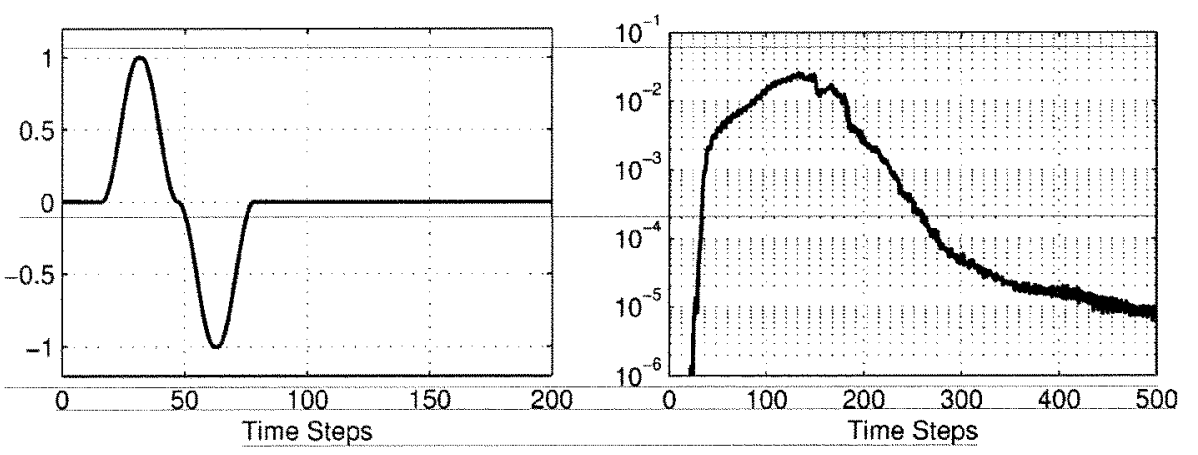

(a)

(b)

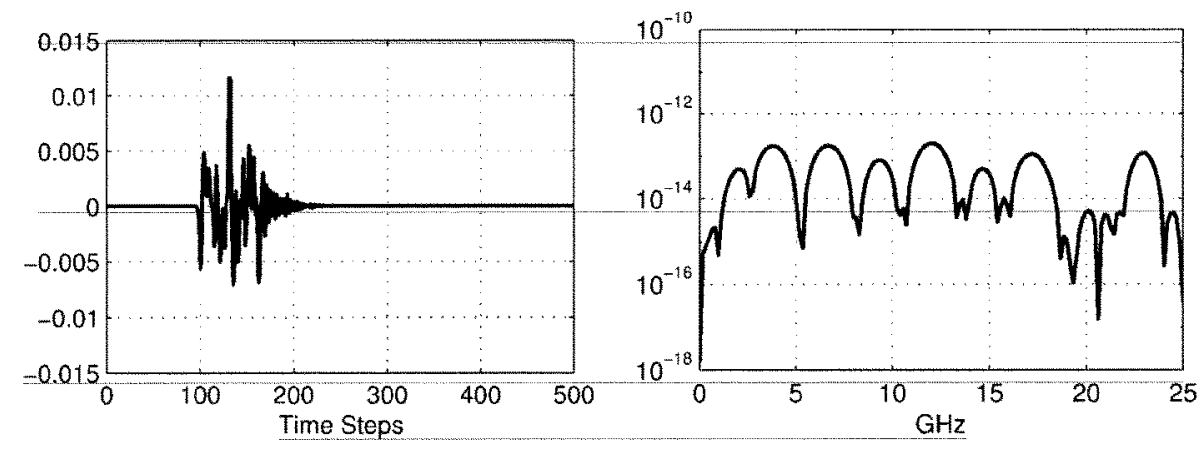

(c)

(d)

Figure 6. Simulation results of a windowed-pulse plane wave. The source grid is sampled with 8-times-higher frequency than the 3-D grid. An anti-decimation digital filter is employed on the windowed source signal. (a) Time variation of the source function. (b) Overall maximum error on $E_{z}$. (c) Error on $E_{z}$ at a particular point. (d) FFT of the error signal displayed in (c). 


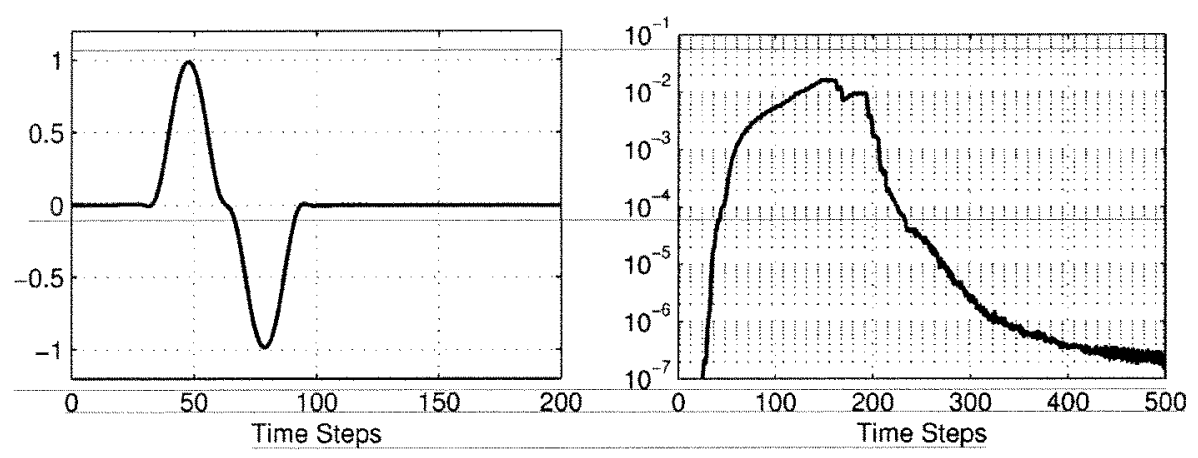

(a)

(b)

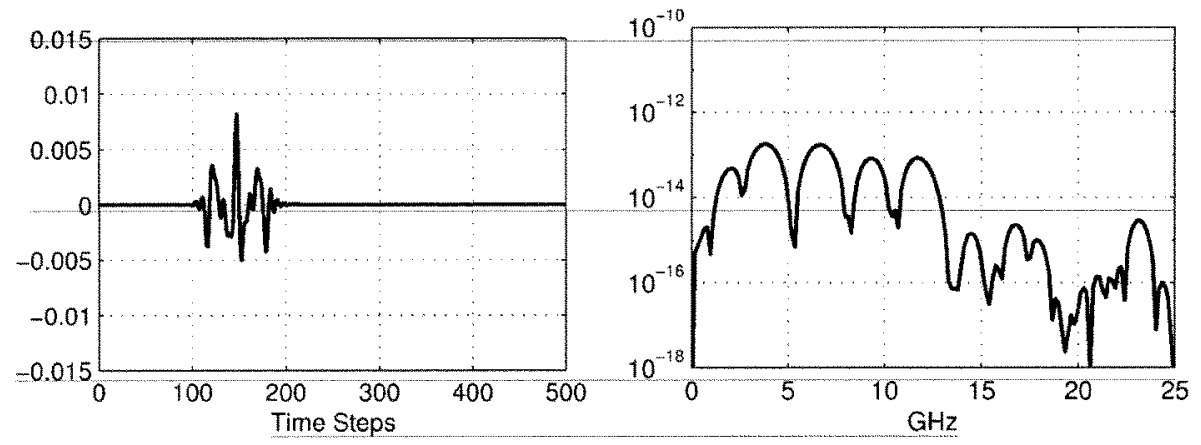

(c)

(d)

Figure 7. Simulation results of a windowed- and filtered-pulse plane wave obtained with an 8times oversampled source grid. An anti-decimation filter and the digital filter in Figure 4 are employed on the windowed source signal. (a) Time variation of the source function. (b) Overall maximum error on $E_{z}$. (c) Error on $E_{z}$ at a particular point. (d) FFT of the error signal displayed in (c).

is not a complex value in the 1-D source grid. However, not all of these frequency components have real wavenumbers in the 3-D grid, and the imaginary parts cause the corresponding frequency components to undergo exponential decay while propagating in the 3-D grid. When the incident-field values are introduced on the total-field/scatteredfield interface, these high-frequency components of the signals in the 1-D and 3-D grids induce an excessive error. By applying a digital lowpass filter with a stop band covering these frequencies, the error due to these high-frequency components of the source grid can be eliminated. In order to demonstrate this decrease, the signal in Figure 6a is passed through a 256-point digital filter with the frequency response shown in Figure 4. Figure 7b demonstrates that, although the filtering process improves the transient-time error level slightly, it primarily decreases the late-time error level in the 3-D FDTD grid. Comparing Figures $6 \mathrm{~d}$ and $7 \mathrm{~d}$, it can be observed that the high-frequency components on the error signal are suppressed by the second filter.

\section{Smooth Pulses}

The frequency-domain representation of a rectangular pulse contains many frequency components that have energies comparable to the center frequency. Since the phase 
velocities of all these frequency components cannot be matched with the 1-D source grid, the dispersion error is increased by using a rectangular pulse. In the preceding three subsections, signal processing techniques, such as oversampling, windowing, and filtering, have been suggested to suppress these dispersion errors. However, if the source function is appropriately chosen, there is no need to window the edges or filter out the high-frequency components to reduce the error. Figure 8 displays the frequency-domain representations of a rectangular and a smooth pulse, which is given by

$$
J(t)=\left[4\left(\frac{t}{\tau}\right)^{3}-\left(\frac{t}{\tau}\right)^{4}\right] e^{-\frac{t}{\tau}} .
$$

In (7), $\tau=1 /\left(4 \pi f_{0}\right)$ and $f_{0}$ is the center frequency of the pulse, which is set as $1 \mathrm{GHz}$. The time dependence of (7) is also displayed in Figure 9a. Figures 8a and 8b demonstrate that the frequency components above $1 \mathrm{GHz}$ exhibit a rapid decay for the pulse in (7), while the same components of the rectangular pulse have much larger energies.

The time dependence of the plane wave is implemented as the function in (7), and the simulation results are given in Figure 9. The amplitude of the smooth function is scaled to equate the value of the $1 \mathrm{GHz}$ spectral component to that of the rectangular pulse of Figure 2a. The maximum error level obtained by (7), shown in Figure 9b, is observed to be approximately 1 order of magnitude below the level obtained with the rectangular pulse, displayed in Figure 2b. However, comparison of Figure 2a to 9a reveals that the peak amplitude of the smooth pulse is more than twice the amplitude of the rectangular pulse. Therefore, the improvement obtained by the smooth pulse is actually larger than one order of magnitude with respect to the rectangular pulse. Comparison of the FFTs of the error signals obtained by the smooth and rectangular pulses, displayed in Figures $9 \mathrm{~d}$ and $2 \mathrm{~d}$, leads to a similar conclusion, i.e., that the error signal obtained with the smooth pulse has attenuated frequency components up to $16.2 \mathrm{GHz}$.

It was shown in the section "Increasing the Sampling Rate of the 1-D Source Grid" that increasing the sampling rate of the source grid reduced the overall error level by decreasing the dispersion error of all frequency components. The same argument holds

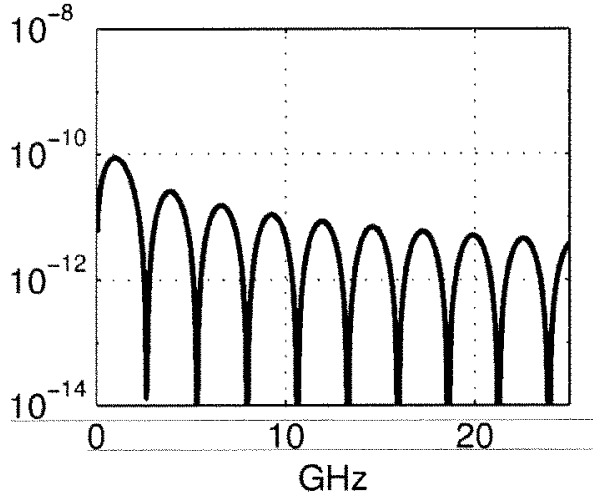

(a)

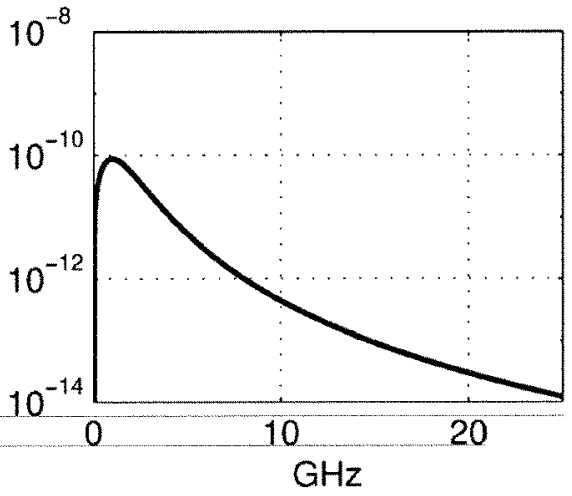

(b)

Figure 8. Frequency-domain representations of (a) the rectangular pulse and (b) the smooth pulse given by (7), both with a center frequency of $1 \mathrm{GHz}$. 


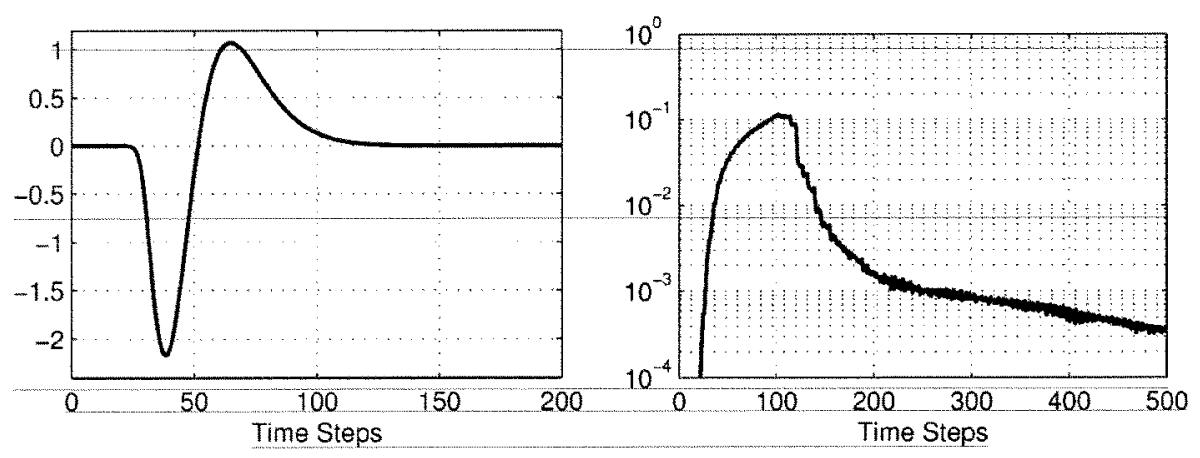

(a)

(b)

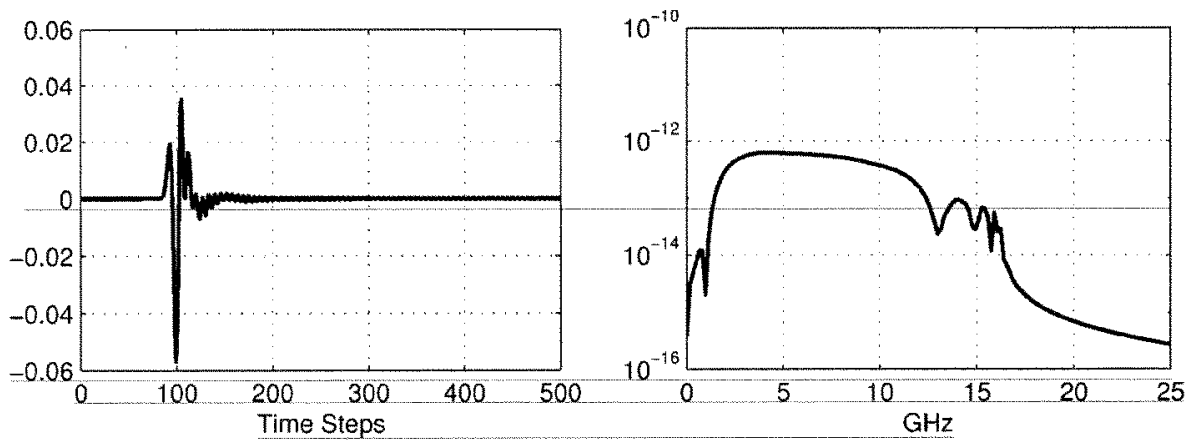

(c)

Figure 9. Simulation results of a transient plane wave with the time dependence in (7) and a center frequency of $1 \mathrm{GHz}$. (a) Time variation of the source function. (b) Overall maximum error on $E_{z}$. (c) Error signal on $E_{z}$ at a particular point. (d) FFT of the error signal displayed in (c).

even when the source function is a narrow-band signal, such as (7), and the dispersion error level is already low. The signal shown in Figure $9 \mathrm{a}$ is sampled with a frequency 8 times higher than the original sampling frequency (approximately $83 \mathrm{GHz}$ ). The oversampled signal is filtered with an anti-decimation filter and fed into the hard source of the source grid. The time dependence of the resultant signal is shown in Figure 10a and the corresponding simulation results are displayed in Figures 10b-10d. Figure 10b illustrates the $50 \%$ decrease in the transient and late-time error levels with a better-sampled source function and 1-D grid. This decrease is further demonstrated by the comparison of the error signals in Figures 9c and 10c, obtained by the smooth pulse fed into the FDTD grid by the standard and 8-times-better-sampled source grid, respectively. The peak-to-peak amplitude of the error signal is almost halved by increasing the spatial and temporal sampling rates of the source grid, due to the suppressed frequency components of the error signal, displayed in Figure 10d.

Comparison of Figure 3 to Figure 9 and of Figure 6 to Figure 10 reveals that the error signals obtained by the smooth pulse are almost twice the signals obtained by the windowed rectangular pulse. Noting that the time-domain amplitude and the $1 \mathrm{GHz}$ spectral value of (7) are both approximately two times larger than the windowed rectangular pulse, it is possible to conclude that this smooth pulse and the windowed rectangular pulse have very similar error performances. 


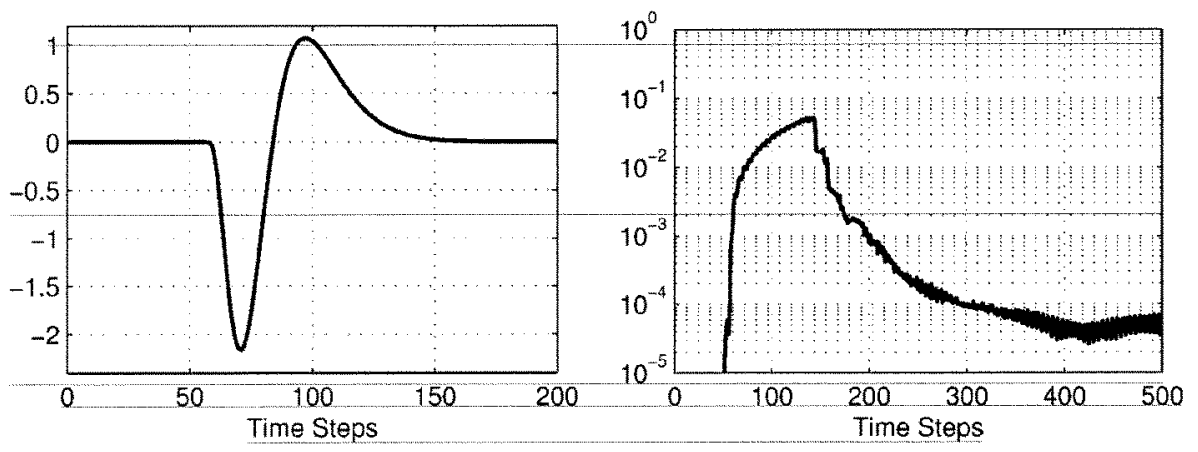

(a)

(b)

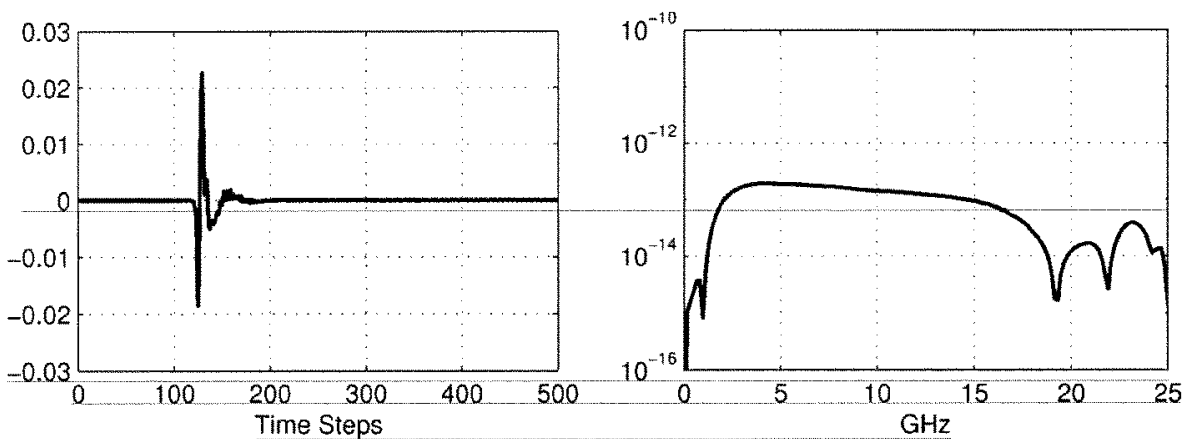

(c)

(d)

Figure 10. Simulation results of the smooth pulse in Figure 9a sampled with an 8-times-higher sampling frequency with respect to the 3-D grid. An anti-decimation digital filter is employed on the smooth pulse to prevent aliasing during the eighth-order decimation. (a) Time variation of the source function. (b) Overall maximum error on $E_{z}$. (c) Error signal on $E_{z}$ at a particular point. (d) FFT of the error signal displayed in (c).

\section{Conclusions}

In this article, the effect of the frequency-domain contents of the multifrequency plane waves on the excitation errors in the FDTD method is demonstrated. The errors due to the numerical dispersion taking place in the FDTD grids are decreased by the application of some SP techniques, which control the frequency content of the source. The simple tools, such as smoothing windows, digital lowpass filters, and polynomial interpolation, have immense effects on the error levels observed in the FDTD computational domain. These effects are demonstrated by simulating the propagation of various multifrequency plane waves in homogeneous media. The leakage from the total-field region to the scattered-field region is regarded as the error, and the SP tools are utilized to decrease this leakage.

\section{References}

Bérenger, J.-P. 1994. A perfectly matched layer for the absorption of electromagnetics waves. J. Comput. Phys. 114:185-200. 
Furse, C. M., S. P. Mathur, \& O. P. Gandhi. 1990. Improvements to the finite-difference timedomain method for calculating the radar cross section of a perfectly conducting target. IEEE Trans. Microwave Theory Tech. 38:919-927.

Gürel, L., \& U. Oğuz. 2000. Signal-processing techniques to reduce the sinusoidal steady-state error in the FDTD method. IEEE Trans. Antennas Propagat. 48:585-593.

Little, J. N., \& L. Shure. 1992. MATLAB signal processing toolbox user's guide. Natick, MA: MathWorks.

Merewether, D. E., R. Fisher, \& F. W. Smith. 1980. On implementing a numeric Huygen's source scheme in a finite difference program to illuminate scattering bodies. IEEE Trans. Nuclear Sci. 27:1829-1833.

Oğuz, U., \& L. Gürel. 1997. Interpolation techniques to improve the accuracy of the incident-wave excitations in the FDTD method. Radio Science 32:2189-2199.

Oğuz, U., L. Gürel, \& O. Arıkan. 1998. An efficient and accurate technique for the incident-wave excitations in the FDTD method. IEEE Trans. Microwave Theory Tech. 46:869-882.

Oppenheim, A. V., \& R. W. Schafer. 1989. Discrete-time signal processing. Englewood Cliffs, NJ: Prentice-Hall.

Parks, T. W., \& C. S. Burrus. 1987. Digital filter design. New York: Wiley.

Schneider, J. B., \& C. L. Wagner. 1999. FDTD dispersion revisited: Faster-than-light propagation. IEEE Microwave and Guided Wave Letters. 9:54-56.

Taflove, A. 1995. Computational electrodynamics: The finite-difference time-domain method. Boston: Artech House.

Taflove, A., \& K. Umashankar. 1983. Radar cross section of general three-dimensional scatterers. IEEE Trans. Electromagn. Compat. 25:433-440.

Umashankar, K. R., \& A. Taflove. 1982. A novel method to analyze electromagnetic scattering of complex objects. IEEE Trans. Electromagn. Compat. 24: 397-405.

van Gennip, G. J. A. 1992. Theory and applications of the 3-dimensional finite-difference timedomain method. Tech. Rep. FEL-92-B190, TNO Physics and Electronics Laboratory.

Yee, K. S. 1966. Numerical solution of initial boundary value problems involving Maxwell's equations in isotropic media. IEEE Trans. Antennas Propagat. 14:302-307. 